SHORT REPORT

\title{
Treatment of stiff person syndrome with rituximab
}

\section{R Baker, M Das, J Isaacs, P R W Fawcett, D Bates}

J Neurol Neurosurg Psychiatry 2005;76:999-1001. doi: 10.1136/jnnp.2004.051144

This case report is about the novel use of the anti-CD20 antibody, rituximab, in the treatment of a 41 year old woman with stiff person syndrome. She was admitted to hospital as an emergency with prolonged and painful extensor spasms affecting the neck and back, arms, and legs. The disease had progressed despite a favourable initial response to conventional treatment with intravenous immunoglobulin and cytotoxics. Treatment with rituximab induced a lasting clinical remission.

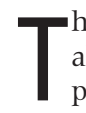
he management of stiff person syndrome is difficult and although success has been reported with the use of plasmapheresis, immunoglobulin, and some cytotoxic agents, most patients continue to follow a relentless and ultimately fatal course. We describe the use of the anti-CD20 antibody, rituximab, in a 41 year old woman with stiff person syndrome. After 15 days of a single treatment she experienced dramatic clinical improvement, cerebrospinal fluid (CSF) antiglutamic acid decarboxylase (anti-GAD) antibodies became undetectable, and electromyograms (EMGs) normalised. Although two months later she required a further course of rituximab, five months after her quality of life is good. Our successful use of a monoclonal anti-B cell antibody in the treatment of stiff person syndrome supports the conclusion that this is a B cell mediated autoimmune disease.

\section{CASE REPORT}

A 41 year old right handed woman with a diagnosis of stiff person syndrome was admitted to hospital as an emergency on 2 January 2004 with prolonged and painful extensor spasms affecting the neck and back, arms, and legs. She had been bed bound for many months due to the condition and was fully dependent upon carers. Her regular medication consisted of baclofen $40 \mathrm{mg}$ thrice daily, dantrolene sodium thrice daily, a fentanyl $25 \mathrm{mg}$ patch twice weekly, and supplemental parenteral diazepam, up to $80 \mathrm{mg}$ per day, and diamorphine, up to $25 \mathrm{mg}$ per day, to manage her severe, painful spasms.

Tone was markedly increased in all muscle groups, predominantly in the axial and proximal limb muscles where there was rigidity. She had a "startle response" which resulted in painful extensor spasms (fig lA). She had previously experienced similar episodes necessitating recurrent admission over the preceding three years.

The patient had been diagnosed as having stiff person syndrome in August 2001. Initial clinical signs included painful spasms of the spinal muscles and deformity of the feet on walking with the appearance of dystonia. Spontaneous involuntary motor unit potentials were demonstrated on EMG (see fig lA) in which the form and magnitude of individual motor units were normal excluding a myopathic process or denervation. She had positive serum anti-GAD (table 1) which supported the diagnosis. When re-examined in 2003, the CSF was positive for anti-GAD antibodies but negative for oligoclonal bands (see table 1).

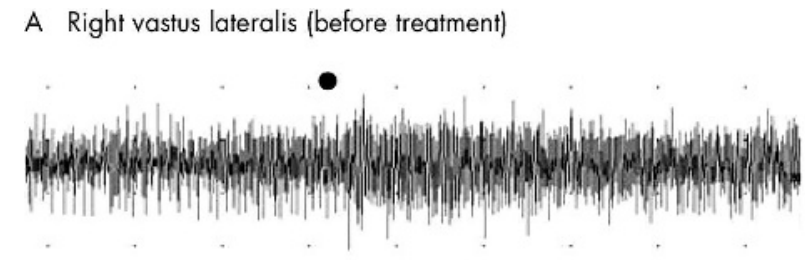

B Right vastus lateralis (after treatment)

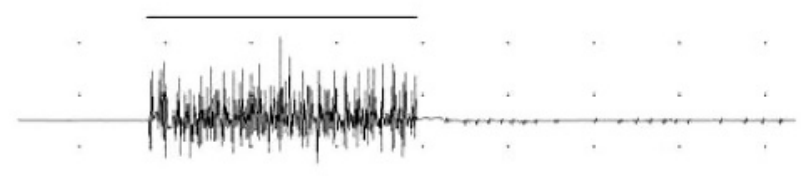

Figure 1 (A) Spontaneous unrectified motor unit activity recorded from the right vastus lateralis at rest (without voluntary contraction). The filled dot indicates the time at which a bystander produced a single loud clap. Note the transiently sustained increase in spontaneous single unit frequency. Time base $20 \mathrm{~s} /$ division and amplitude $2 \mathrm{mV} /$ division. (B) Spontaneous unrectified motor unit activity recorded from the right vastus lateralis at rest after treatment. The solid bar indicates a voluntary contraction. Time base $10 \mathrm{~s}$ and amplitude $1 \mathrm{mV}$.

The past medical history included a hysterectomy and bilateral salpingo-oophorectomy for premenstrual dysphoric disorder and leucopenia secondary to IgM antigranulocyte antibodies. Antigastric parietal cell, antimitochondrial, antismooth muscle, anti-dsDNA, anti-ENA, antineutrophil cytoplasmic, and antiendomysial antibodies, and rheumatoid factor, and antinuclear antibodies were negative. She did not smoke tobacco or drink alcohol. Her family history was notable only for ischaemic heart disease on the maternal side.

Various antispastic agents were tried over the years following diagnosis, including tizanidine, piracetam, phenobarbital, and local botulinum toxin (Botox; Allergen Ltd, Crenex, High Wycombe, Bucks) injections. Only diazepam, baclofen, and dantrolene were tolerated and provided subjective benefit.

Various disease modifying treatments were tried. She received seven courses of intravenous immunoglobulin from November 2001 to May 2003; there was some functional improvement following the initial two courses but not subsequently. Treatment with plasmapheresis was considered but could not be implemented due to religious conviction (the patient is a Jehovah's Witness).

Cyclophosphamide was introduced in May 2002 with some benefit but had to be withdrawn because of severe neutropenia. Mycophenolate mofetil was started in May 2003 at a dose of $500 \mathrm{mg}$ twice daily and was initially effective but later had to be escalated to $1 \mathrm{~g}$ twice daily then to $1.5 \mathrm{~g}$ twice daily in November 2003 due to recurrence of symptoms. It was discontinued in December 2003 because

Abbreviation: anti-GAD, antiglutamic acid decarboxylase 
Table 1 Results of blood and cerebrospinal fluid (CSF) analysis of the patient

\begin{tabular}{|c|c|c|c|}
\hline Date & $\begin{array}{l}\text { Oligoclonal } \\
\text { bands }\end{array}$ & $\begin{array}{l}\text { Serum anti-GAD } \\
\text { (units/ml) } \\
\text { (normal range } \\
<1 \text { ) }\end{array}$ & $\begin{array}{l}\text { CSF anti-GAD } \\
\text { (units/ml) }\end{array}$ \\
\hline $30 \mathrm{Jul} 2001$ & - & Negative & \\
\hline 07 Aug 2001 & Negative & - & - \\
\hline 16 May 2002 & - & 1.1 & - \\
\hline 10 Dec 2002 & - & 0.5 & - \\
\hline 31 Oct 2003 & - & 0.2 & - \\
\hline 19 Nov 2003 & Negative & 0.1 & 3.2 \\
\hline 09 Jan 2004 & \multicolumn{3}{|c|}{ Intravenous rituximab $\left(375 \mathrm{mg} / \mathrm{m}^{2}\right)$ administered } \\
\hline $26 \operatorname{Jan} 2004$ & - & 0.2 & 0 \\
\hline 02 Apr 2004 & Negative & 0.3 & 0 \\
\hline \multicolumn{4}{|c|}{ Intravenous rituximab $\left(375 \mathrm{mg} / \mathrm{m}^{2}\right)$ per week $\times 4$} \\
\hline 30 Apr 2004 & Negative & 0.3 & 0 \\
\hline
\end{tabular}

the dose was no longer controlling the disease. She was then given once weekly infusions of intrathecal hydrocortisone $(500 \mathrm{mg}$ ) for four weeks in an attempt to suppress any intrathecal clone of antibody producing cells. This treatment produced short lived benefit and allowed the patient to return home for Christmas, before readmission in January when her symptoms deteriorated.

Rituximab was administered intravenously on 9 January 2004 at a dose of $375 \mathrm{mg} / \mathrm{m}^{2}$. Rituximab is an anti-CD20 chimeric human mouse monoclonal antibody that specifically depletes mature B lymphocytes by binding irreversibly and destroying them by apoptosis, antibody dependent cell mediated cytotoxicity (ADCC), and complement mediated lysis. Fifteen days after the infusion the stiffness began to resolve and the patient was able to sit up and shower herself for the first time in more than two years. Figure 1B illustrates the normalisation of the right vastus lateralis EMG following this treatment. The CSF was re-examined on the day of discharge, 17 days after treatment with rituximab, and no anti-GAD antibodies were detectable. One month after discharge she was stable and symptoms were easily managed by small doses of oral benzodiazepines and analgesia.

Symptoms began to reappear after the sixth week and she was readmitted and given a four week course of intravenous rituximab in the same dosage at weekly intervals and mycophenolate mofetil was reintroduced at a dose of $\mathrm{l} \mathrm{g}$ twice daily. Her condition again improved after 14 days and she was discharged at the end of the course, at which time she was able to stand and walk with help, sit, and shower.

\section{DISCUSSION}

Stiff person syndrome is a rare progressive autoimmune disease of the central nervous system (CNS). The diagnosis is made, after exclusion of other known pathologies, primarily on clinical criteria of stiffness, predominantly in axial muscles, with co-contraction of agonist and antagonist muscles and paroxysmal spasms. The diagnosis is supported by investigations including neurophysiological examination, which demonstrates spontaneous motor unit activity at rest simultaneously from agonist and antagonist muscles, and immunological examination, which reveals high serum and CSF titres of anti-GAD antibodies. In addition to these antibodies, antiamphiphysin antibodies, ${ }^{1}$ antigephyrin and anti-Ri antibodies ${ }^{2}$ are often found in paraneoplastic stiff person syndrome.

The mechanism of stiffness in stiff person syndrome is assumed to be a consequence of the interaction between the anti-GAD antibody and the CNS. GAD is the rate limiting enzyme in the production of $\gamma$-aminobutyric acid (GABA), which is the most abundant inhibitory neurotransmitter within the CNS. Anti-GAD antibody is known to block the synthesis of GABA in vitro, ${ }^{3}$ but whether there is internalisation of anti-GAD antibody and inhibition of GABA synthesis, or whether the antibody attacks and destroys interneurones expressing surface GAD is not clear. However, it is generally accepted that the stiff person syndrome phenotype is the consequence of the functional impairment of inhibitory interneurones as evidenced by the effectiveness of the various GABAergic agents used in the symptomatic treatment of stiff person syndrome.

Electrophysiological methods have been employed to investigate more precisely the locus of GABAergic dysfunction within the CNS in stiff person syndrome, and excitability changes have been described at the level of cortex and spinal cord, ${ }^{4}$ and brainstem..$^{5-7}$ Increased excitability within the brainstem and cortex might explain tonic muscle stiffness to some extent but is more likely to explain the presence of the startle response often described in stiff person syndrome. Post mortem histopathological reports have described reduced numbers of GABAergic cells within the cerebellum and a reduction in the size of Renshaw's cells within the spinal cord. ${ }^{8}$

Symptomatic treatment relies upon GABA enhancing agents, and as a putative B cell mediated disease, immunomodulatory therapies have been tried with varying success. The most compelling evidence for immunomodulatory treatment comes from a placebo controlled crossover trial of high dose intravenous immunoglobulin in 16 patients with stiff person syndrome. ${ }^{9}$ In addition to significant improvement in objective scores of function there was a significant reduction in anti-GAD in the treatment arm.

Further evidence that stiff person syndrome is B cell mediated disease is its strong association with other autoimmune diseases. Although our patient had none of the more recognised autoimmune diseases, she did have autoimmune granulocytopenia. Antineutrophil antibodies have been described in association with both systemic lupus erythematosus $(\mathrm{SLE})^{10}$ and idiopathic thrombocytopenic purpura. ${ }^{11}$

Rituximab has been used with success both in the treatment of other autoimmune conditions (for review see Silverman and Weisman ${ }^{12}$ ), including rheumatoid arthritis and SLE, and combined with cytotoxic chemotherapeutic agents in the treatment of non-Hodgkin's lymphoma. ${ }^{13}$ Rituximab specifically targets cells expressing the surface antigen CD20 and therefore depletes mature B lymphocytes, but spares plasma cells and early pre-B cells, which do not express CD20. Some trials of rituximab therapy in autoimmune disease (e.g. idiopathic thrombocytopenic purpura) and non-Hodgkin's lymphoma (NHL) have failed to show clinical improvement. In autoimmune disease, this is usually because of the low doses used in the initial trials, and in NHL, this has been attributed to reduced expression of CD20 in lymphoma cells in advanced disease. ${ }^{12}$ With long term treatment there are the theoretical concerns of sensitisation and diminished effectiveness.

This is the first report of the successful use of a monoclonal anti-B cell antibody in the treatment of stiff person syndrome. The rapid decline of intrathecal anti-GAD following treatment suggests the successful targeting and elimination of autoantibody producing B cells within the CNS. The association of anti-GAD decline with clinical improvement supports the suggestion that SPS is a B cell mediated autoimmune disease. The rapidity and extent of recovery following treatment might suggest that there is minimal neuronal destruction, and therefore that the syndrome is due to functional inhibition of GABAergic neurones by autoantibodies. 


\section{Authors' affiliations}

M R Baker, M Das, D Bates, Department of Neurology, Royal Victoria Infirmary, Newcastle upon Tyne, UK

P R W Fawcett, Department of Neurophysiology, Royal Victoria Infirmary, Newcastle upon Tyne, UK

J Isaacs, Department of Rheumatology, Royal Victoria Infirmary,

Newcastle upon Tyne, UK

Competing interests: none declared

Correspondence to: Prof D Bates, Department of Neurology, Royal Victoria Infirmary Queen Victoria Road, Newcastle upon Tyne NE1 4LP, UK; david.bates@ncl.ac.uk

Received 3 August 2004

Revised version received 24 September 2004

Accepted 26 September 2004

\section{REFERENCES}

1 Rosin L, DeCamelli P, Butler M, et al. Stiff-man syndrome in a woman with breast cancer: an uncommon central nervous system paraneoplastic syndrome Neurology 1998;50:94-8.

2 McCabe DJH, Turner NC, Chao D, et al. Paraneoplastic "stiff person syndrome" with metastatic adenocarcinoma and anti-Ri antibodies. Neurology 2004;62:1402-4
3 Dinkel K, Meinck HM, Jury KM, et al. Inhibition of gamma-aminobutyric acid synthesis by glutamic acid decarboxylase autoantibodies in stiff-man syndrome. Ann Neurol 1998:44:194-201.

4 Sandbrink F, Syed NA, Fujii MD, et al. Motor cortex excitability in stiff-person syndrome. Brain 2000;123:2231-9.

5 Matsumoto JY, Caviness JN, McEvoy KM. The acoustic startle reflex in stiffman syndrome. Neurology 1994;44:1952-5.

6 Meinck H-M, Ricker K, Hulser PJ, et al. Stiff man syndrome: clinical and laboratory findings in eight patients. J Neurol 1994;241:157-66.

7 Meinck H-M, Ricker K, Hulser PJ, et al. Stiff man syndrome: neurophysiological findings in eight patients J Neurol 1995:242:134-42.

8 Warich-Kirches M, Von Bossanyi P, Treuheit T, et al. Stiff-man syndrome: possible autoimmune aetiology targeted against GABA-ergic cells. 1997; 16:214-19

9 Dalakas OM, Fujii $M$, Li $M$, et al. High-dose intravenous immune globulin for stiff-person syndrome. New Engl J Med 2001;345:1870-6.

10 Hadley AG, Byron MA, Chapel HM, et al. Anti-granulocyte opsonic activity in sera from patients with lupus erythematosus $\mathrm{Br} J$ Haemato $1987 ; 65: 61-5$

11 Vlasveld LT, deHaas M, Ermens AA, et al. G-CSF-induced decrease of the anti-granulocyte autoantibodies in a patient with autoimmune granulocyłopenia. Ann Haematol 1997;75:59-64

12 Silverman GJ, Weisman S. Rituximab therapy and autoimmune disorders. Arthritis Rheum 2003;48:1484-92.

13 Avivi I, Robinson S, Goldstone A. Clinical use of rituximab in haematological malignancies. Br J Cancer 2003;89:1389-94. 